\title{
A New Approach on Transforms: Formable Integral Transform and Its Applications
}

\author{
Rania Zohair Saadeh * ${ }^{(\mathbb{B}}$ and Bayan fu'ad Ghazal
}

check for

updates

Citation: Saadeh, R.Z.; Ghazal, B.f. A

New Approach on Transforms:

Formable Integral Transform and Its

Applications. Axioms 2021, 10, 332.

https://doi.org/10.3390/

axioms10040332

Academic Editor: Palle E.T. Jorgensen

Received: 25 September 2021

Accepted: 5 November 2021

Published: 1 December 2021

Publisher's Note: MDPI stays neutral with regard to jurisdictional claims in published maps and institutional affiliations.

Copyright: (c) 2021 by the authors. Licensee MDPI, Basel, Switzerland. This article is an open access article distributed under the terms and conditions of the Creative Commons Attribution (CC BY) license (https:/ / creativecommons.org/licenses/by/ $4.0 /)$.
Department of Mathematics, Faculty of Science, Zarqa University, Zarqa 13110, Jordan; 20209128@zu.edu.jo

* Correspondence: rsaadeh@zu.edu.jo

\begin{abstract}
In this paper, we introduce a new integral transform called the Formable integral transform, which is a new efficient technique for solving ordinary and partial differential equations. We introduce the definition of the new transform and give the sufficient conditions for its existence. Some essential properties and examples are introduced to show the efficiency and applicability of the new transform, and we prove the duality between the new transform and other transforms such as the Laplace transform, Sumudu transform, Elzaki transform, ARA transform, Natural transform and Shehu transform. Finally, we use the Formable transform to solve some ordinary and partial differential equations by presenting five applications, and we evaluate the Formable transform for some functions and present them in a table. A comparison between the new transform and some well-known transforms is made and illustrated in a table.
\end{abstract}

Keywords: Laplace transform; Shehu transform; Natural transform; ARA transform; Fourier transform; Elzaki transform; Sumudu transform; ordinary differential equation; partial differential equation; integral transform

\section{Introduction}

Differential equations represent a field of mathematics that has great applications in science, since they are used in mathematical modeling [1-9] and hence aid in finding solutions in physical and engineering problems involving functions of one or several variables, such as the propagation of heat or sound, fluid flow, elasticity, electrostatics, electrodynamics, etc.

For decades, methods for solving differential equations have been important subjects for researchers, [10-19] because of their important applications in various fields of science. The technique of using integral transforms has proved its efficiency and applicability in solving ordinary and partial differential equations.

For the function $g(t)$ and $t \in(-\infty, \infty)$, the integral transform is obtained by computing the improper integral

$$
£[g(t)](s)=\int_{-\infty}^{\infty} k(s, t) g(t) d t
$$

where $k(s, t)$ is called the kernel of the integral transform and $s$ is the variable of the transform, which might be real or complex number and is independent of the variable $t$. The theory of integral transforms goes back to the work of P.S. Laplace in $1780[19,20]$ and Fourier in 1822. Recently, the idea of using integral transforms in solving differential equations and integral equations has been commonly used by many researchers in the literature [21-30].

The Laplace transform is defined as:

$$
£[g(t)]=G(s)=\int_{0}^{\infty} \exp (-s t) g(t) d t
$$


and it shows high efficiency in solving a class of differential equations. By replacing the variable $s$ by $i w$ and multiplying Equation (2) by $\frac{1}{\sqrt{ } 2 \pi}$ we obtain the well-known Fourier integral transform, defined as

$$
F[g(t)]=g(w)=\frac{1}{\sqrt{ } 2 \pi} \int_{-\infty}^{\infty} \exp (-i w t) g(t) d t
$$

These transforms are basic in the study of integral transforms, but the difference between them is that the Laplace transform is applicable for both stable and unstable systems, but the Fourier transform is only defined for stable systems.

For many years, the theory of integral transforms has been very widely studied in the mathematical literature, and many researchers have investigated new transforms such as the z-transform [29], the Mellin integral transform [30], the Laplace-Carson transform [31] and the Hankel transform [32,33].

The Sumudu integral transform [34] was introduced in 1993. It showed applicability in solving real-life problems and was used for solving differential equations. The Sumudu integral transform is defined as:

$$
S[g(t)](u)=G(u)=\frac{1}{u} \int_{0}^{\infty} \exp \left(\frac{-t}{u}\right) g(t) d t .
$$

In 2008, Belgacem and Silambarasan introduced the Natural transform, as follows:

$$
N^{+}[g(t)](s, u)=R(s, u)=\frac{1}{u} \int_{0}^{\infty} \exp \left(\frac{-s t}{u}\right) g(t) d t, \quad s, u>0 .
$$

The Elzaki integral transform was obtained in 2011, with the definition

$$
E[g(t)](u)=T(u)=u \int_{0}^{\infty} \exp \left(\frac{-t}{u}\right) g(t) d t .
$$

This is closely related to the Laplace and Sumudu integral transforms. The Shehu integral transform is given by

$$
\mathbb{S}[g(t)](s, u)=V(s, u)=\int_{0}^{\infty} \exp \left(\frac{-s t}{u}\right) g(t) d t, s, u>0 .
$$

This also shows the ability to solve a class of differential equations and, combined with other numerical methods of solving differential equations, to offer a new approach in dealing with fractional differential equations.

In 2020, the ARA transform was introduced by Saadeh et al. and was implemented to solve a wide range of fractional ordinary and partial differential equations.

The ARA integral transform is given by

$$
\mathcal{G}_{n}[g(t)](s)=G(n, s)=s \int_{0}^{\infty} t^{n-1} \exp (-s t) g(t) d t, s>0 .
$$

Recently, the above transforms and others have been combined with other analytical methods in mathematics to solve a wide range of linear and nonlinear fractional and ordinary differential equations, and other methods are shown in.

In this paper, we propose a new integral transform called the Formable transform. We introduce the definition and some properties of the new transform in Section 2. The 
dualities between the Formable and other transforms are illustrated in Section 3 with some examples. In Section 4, we apply the Formable transform in some ordinary and partial differential equations to show its efficiency and accuracy through applications. Finally, the values of the Formable transform for some special functions are presented in a table.

\section{Definitions and Theorems}

In this section we introduce the definition of the new transform called the Formable transform, together with some theorems and properties of the new transform.

Definition 1. The Formable integral transform of a function $g(t)$ of exponential order is defined over the set of functions

$$
W=\left\{g(t): \exists N \in(0, \infty), \tau_{i}>0 \text { for } i=1,2,|g(t)|<N \exp \left(\frac{t}{\tau_{i}}\right), \text { if } \mathrm{t} \in[0, \infty)\right\},
$$

in the following form:

$$
R[g(t)]=B(s, u)=s \int_{0}^{\infty} \exp (-s t) g(u t) d t
$$

This is equivalent to

$$
\begin{gathered}
R[g(t)]=\frac{s}{u} \int_{0}^{\infty} \exp \left(\frac{-s t}{u}\right) g(t) d t \\
R[g(t)]=\frac{s}{u} \lim _{x \rightarrow \infty} \int_{0}^{x} \exp \left(\frac{-s t}{u}\right) g(t) d t, \quad \mathrm{~s}>0, u>0
\end{gathered}
$$

where $s$ and $u$ are the Formable transform's variables, $x$ is a real number and the integral is taken along the line $t=x$. A function $g(t)$ is said to be of exponential order $c$ if there exist constants $M$ and $T$ such that $|g(t)| \leq M e^{c t}$ for all $t \geq T$. Here, we mention that we chose the name "Formable" for this new transform because of its flexibility in solving ordinary and partial differential equations. In addition, it has a duality with other wellknown transforms that will be considered later. To show the applicability of the Formable transform, we compute the transform for several functions in Section 3. We compare the results with other values from some well-known transforms and illustrate them in a table in the Appendix A.

The inverse Formable transform of a function $g(t)$ is given by

$$
R^{-1}[B(s, u)]=g(t)=\frac{1}{2 \pi i} \int_{c-i \infty}^{c+i \infty} \frac{1}{s} \exp \left(\frac{s t}{u}\right) B(s, u) d s .
$$

That is, from the definition of the Fourier transform, we know

$$
F[g(t)]=F(w)=\frac{1}{\sqrt{2 \pi}} \int_{-\infty}^{\infty} e^{-i w t} g(t) d t F^{-1}[F(w)]=g(t)=\frac{1}{\sqrt{2 \pi}} \int_{-\infty}^{\infty} e^{i w t} F(w) d w .
$$

Then

$$
\begin{aligned}
g(t)= & \frac{1}{\sqrt{2 \pi}} \int_{-\infty}^{\infty} e^{i w t}\left[\frac{1}{\sqrt{2 \pi}} \int_{-\infty}^{\infty} e^{-i w t} g(t) d t\right] d w \\
& =\frac{1}{2 \pi} \int_{-\infty}^{\infty} e^{i w t}\left[\int_{-\infty}^{\infty} e^{-i w t} g(t) d t\right] d w
\end{aligned}
$$

where $g(t)$ is a function defined on the domain $(-\infty, \infty)$, so that for $t \in(-\infty, 0)$ we assume that $g(t)=0$. Hence for $\mathrm{t}>0$, let $g(t)=g(t) u(\mathrm{t}) e^{-c t}$, where $u(t)$ is the unit step function and $c$ is any constant, so that Equation (11) becomes

$$
g(t) u(t) e^{-c t}=\frac{1}{2 \pi} \int_{-\infty}^{\infty} e^{i w t}\left[\int_{0}^{\infty} e^{-(c+i w) t} g(t) d t\right] d w .
$$


Multiplying both sides of Equation (12) by $e^{c t}$, we obtain

$$
g(t) u(t)=\frac{1}{2 \pi} \int_{-\infty}^{\infty} e^{(c+i w) t}\left[\int_{0}^{\infty} e^{-(c+i w) t} g(t) d t\right] d w
$$

Substituting $\frac{s}{u}=c+i \omega, \frac{d s}{u}=i d w$ and $d w=\frac{1}{i u} d s$ in Equation (13), we obtain

$g(t) u(t)=\frac{1}{2 \pi i} \int_{c-i \infty}^{c+i \infty} e^{\frac{s t}{u}} \frac{1}{u}\left[\int_{0}^{\infty} e^{-\frac{s t}{u}} g(t) d t\right] d s=\frac{1}{2 \pi i} \int_{c-i \infty}^{c+i \infty} \frac{1}{s} e^{\frac{s t}{u}}\left[\frac{s}{u} \int_{0}^{\infty} e^{-\frac{s t}{u}} g(t) d t\right] d s=\frac{1}{2 \pi i} \int_{c-i \infty}^{c+i \infty} \frac{1}{s} e^{\frac{s t}{u}} B(s, u) d s$.

Defining $g(t)$ on $(0, \infty)$, we obtain

$$
g(t)=\frac{1}{2 \pi i} \int_{\mathcal{c}-i \infty}^{c+i \infty} \frac{1}{s} e^{\frac{s t}{u}} B(s, u) d s
$$

Hence,

$$
R^{-1}[B(s, u)]=\frac{1}{2 \pi i} \int_{c-i \infty}^{c+i \infty} \frac{1}{s} \exp \left(\frac{s t}{u}\right) B(s, u) d s,
$$

and

$$
R^{-1}[R(g(t))]=g(t)
$$

Theorem 1. Sufficient conditions for the existence of the Formable transform.

If the function $g(t)$ is a piecewise continuous function in every finite interval $t \in[0, \alpha]$ and is of exponential order $\beta$ for $t>\beta$, then the Formable transform $B(s, u)$ of $g(t)$ exists.

Proof. Let $\alpha$ be any positive number, then we have

$$
B(s, u)=\frac{s}{u} \int_{0}^{\infty} \exp \left(\frac{-s t}{u}\right) g(t) d t=\frac{s}{u} \int_{0}^{\alpha} \exp \left(\frac{-s t}{u}\right) g(t) d t+\frac{s}{u} \int_{\alpha}^{\infty} \exp \left(\frac{-s t}{u}\right) g(t) d t
$$

Since the function $g(t)$ is a piecewise continuous function in every finite interval $[0, \alpha]$, the integral $\frac{s}{u} \int_{0}^{\alpha} \exp \left(\frac{-s t}{u}\right) g(t) d t$ exists, and since $g(t)$ is of exponential order $\beta$ we have

$$
\begin{aligned}
\left|\frac{s}{u} \int_{\alpha}^{\infty} \exp \left(\frac{-s t}{u}\right) g(t) d t\right| & \leq\left|\frac{s}{u}\right| \int_{\alpha}^{\infty}\left|\exp \left(\frac{-s t}{u}\right) g(t)\right| d t \\
& =\frac{s}{u}\left|\int_{\alpha}^{\infty} \exp \left(\frac{-s t}{u}\right)\right| g(t) \mid d t \\
& \leq \frac{s}{u} \mid \int_{\alpha}^{\infty} \exp \left(\frac{-s t}{u}\right) N \exp (\beta t) d t \\
& =\left|\frac{s}{u}\right| N \int_{\alpha}^{\infty} \exp \left(-t\left(\frac{s}{u}-\beta\right)\right) d t \\
& \leq \frac{s}{u} N \int_{0}^{\infty} \exp \left(-t\left(\frac{s}{u}-\beta\right)\right) d t \\
& =\frac{s}{u} N \lim _{x \rightarrow \infty}\left[-\frac{\exp \left(-t\left(\frac{s}{u}-\beta\right)\right)}{\frac{s}{u}-\beta}\right]_{t=0}^{x} \\
& =\frac{s}{u} N\left(\frac{1}{\frac{s}{u}-\beta}\right) \\
& =s N /(s-\beta u) .
\end{aligned}
$$

The proof is complete. Now, we introduce some basic properties and results concerning the Formable transform which enable us to solve more applications via the transform.

Property 1 (linearity property). Let $\alpha g_{1}(t)$ and $\beta g_{2}(t)$ be two functions in a set $W$, then $\left(\alpha g_{1}(t)+\beta g_{2}(t)\right) \in W$, where $\alpha$ and $\beta$ are nonzero arbitrary constants, and

$$
R\left[\alpha g_{1}(t)+\beta g_{2}(t)\right]=\alpha R\left[g_{1}(t)\right]+\beta R\left[g_{2}(t)\right] .
$$


Proof of Property 1. Using the definition of the Formable transform, we have

$$
\begin{aligned}
& R\left[\alpha g_{1}(t)+\beta g_{2}(t)\right]=\frac{s}{u} \int_{0}^{\infty} \exp \left(\frac{-s t}{u}\right)\left(\alpha g_{1}(t)+\beta g_{2}(t)\right) d t \\
= & \frac{s}{u} \int_{0}^{\infty} \exp \left(\frac{-s t}{u}\right) \alpha g_{1}(t) d t+\frac{s}{u} \int_{0}^{\infty} \exp \left(\frac{-s t}{u}\right) \beta g_{2}(t) d t \\
= & \alpha \frac{s}{u} \int_{0}^{\infty} \exp \left(\frac{-s t}{u}\right) g_{1}(t) d t+\beta \frac{s}{u} \int_{0}^{\infty} \exp \left(\frac{-s t}{u}\right) g_{2}(t) d t \\
= & \alpha R\left[g_{1}(t)\right]+\beta R\left[g_{2}(t)\right] .
\end{aligned}
$$

The proof is complete.

Property 2 (change of scale). Let the function $g(\alpha t)$ be in the set $W$, where $\alpha$ is an arbitrary constant, then

$$
R[g(\alpha t)]=B\left(\frac{S}{\alpha}, u\right)=B(s, \alpha u)
$$

\section{Proof of Property 2.}

$$
R[g(\alpha t)]=\frac{s}{u} \int_{0}^{\infty} \exp \left(\frac{-s t}{u}\right) g(\alpha t) d t
$$

Substituting $\delta=\alpha t$ in Equation (17) we have

$$
\begin{aligned}
R[g(\alpha t)] & =\frac{s}{u} \int_{0}^{\infty} \exp \left(\frac{-s \delta}{u \alpha}\right) g(\delta) \frac{d \delta}{\alpha} \\
& =\frac{s}{\alpha u} \int_{0}^{\infty} \exp \left(\frac{-s \delta}{u \alpha}\right) g(\delta) d \delta \\
& =B\left(\frac{s}{\alpha}, u\right) \\
& =B(s, \alpha u) .
\end{aligned}
$$

Property 3 (Formable transform of the derivative). If the function $g^{(n)}(t)$ is the $n$-th derivative of the function $g(t)$, where $g^{(n)}(t) \in W$, for $n=0,1,2, \ldots$ with respect to $t$, then

$$
R\left[g^{(n)}(t)\right]=\frac{s^{n}}{u^{n}} B(s, u)-\sum_{k=0}^{n-1}\left(\frac{s}{u}\right)^{n-k} g^{(k)}(0) .
$$

Proof of Property 3. For $n=1$, we have

$$
\begin{aligned}
R\left[g^{\prime}(t)\right] & =\frac{s}{u} \int_{0}^{\infty} \exp \left(\frac{-s t}{u}\right) g^{\prime}(t) d t \\
& =\frac{s}{u}\left[\lim _{\alpha \rightarrow \infty}\left[\exp \left(\frac{-s t}{u}\right) g(t)\right]_{t=0}^{\alpha}+\frac{s}{u} \int_{0}^{\infty} \exp \left(\frac{-s t}{u}\right) g(t) d t\right] \\
& =\frac{s}{u}[-g(0)+B(s, u)] .
\end{aligned}
$$

Thus

$$
R\left[g^{\prime}(t)\right]=\frac{s}{u} B(s, u)-\frac{s}{u} g(0)
$$

Assuming that Equation (18) is true for $n=k$, then we show that it is true for $n=k+1$, by using the fact that in Equation (19) we have

$$
\begin{aligned}
R\left[g^{(\mathrm{k}+1)}(\mathrm{t})\right] & =R\left[\left(g^{(k)}(\mathrm{t})\right)^{\prime}\right] \\
& =\frac{s}{u} R\left[g^{(k)}(\mathrm{t})\right]-\frac{s}{u} g^{(k)}(0) \\
& =\frac{s}{u}\left[\frac{s^{k}}{u^{k}} B(s, u)-\sum_{i=0}^{k-1}\left(\frac{s}{u}\right)^{k-i} g^{(i)}(0)\right]-\frac{s}{u} g^{(k)}(0) \\
& =\frac{s^{k+1}}{u^{k+1}} B(s, u)-\sum_{i=0}^{k}\left(\frac{s}{u}\right)^{k+1-i} g^{(i)}(0)
\end{aligned}
$$

This implies that Equation (17) holds for $n=k+1$, so the proof is complete. 
The following important properties are obtained using the Leibniz rule and Equation (18):

$$
\begin{aligned}
\text { (i) } R\left[\frac{\partial g(x, t)}{\partial x}\right] & =\frac{s}{u} \int_{0}^{\infty} \exp \left(\frac{-s t}{u}\right) \frac{\partial g(x, t)}{\partial x} d t \\
& =\frac{\partial}{\partial x}\left[\frac{s}{u} \int_{0}^{\infty} \exp \left(\frac{-s t}{u}\right) g(x, t) d t\right]=\frac{\partial}{\partial x}[B(x, s, u)] . \\
\text { (ii) } R\left[\frac{\partial^{2} g(x, t)}{\partial x^{2}}\right] & =\frac{s}{u} \int_{0}^{\infty} \exp \left(\frac{-s t}{u}\right) \frac{\partial^{2} g(x, t)}{\partial x^{2}} d t \\
& =\frac{\partial^{2}}{\partial x^{2}}\left[\frac{s}{u} \int_{0}^{\infty} \exp \left(\frac{-s t}{u}\right) g(x, t) d t\right]=\frac{\partial^{2}}{\partial x^{2}}[B(x, s, u)] . \\
\text { (iii) } R\left[\frac{\partial^{n} g(x, t)}{\partial x^{n}}\right] & =\frac{s}{u} \int_{0}^{\infty} \exp \left(\frac{-s t}{u}\right) \frac{\partial^{n} g(x, t)}{\partial x^{n}} d t \\
& =\frac{\partial^{n}}{\partial x^{n}}\left[\frac{s}{u} \int_{0}^{\infty} \exp \left(\frac{-s t}{u}\right) g(x, t) d t\right] \\
& =\frac{\partial^{n}}{\partial x^{n}}[B(x, s, u)] .
\end{aligned}
$$

Property 4 (Formable transform of the convolution). If $F(s, u)$ and $G(s, u)$ are the Formable transforms of the functions $f(t)$ and $g(t)$, respectively, then

$$
R[f(t) * g(t)]=\frac{u}{s} F(s, u) G(s, u),
$$

where $f(t) * g(t)$ is the convolution of the functions $f(t)$ and $g(t)$ defined by

$$
f(t) * g(t)=\int_{0}^{t} f(\tau) g(t-\tau) d \tau
$$

Proof of Property 4. Using the definition of the Formable transform in Equation (9), we obtain

$$
\begin{aligned}
R[f(t) * g(t)] & =s \int_{0}^{\infty} \exp (-s t)(f * g)(u t) d t \\
& =s \int_{0}^{\infty} \exp (-s t) \int_{0}^{u t} f(\tau) g(u t-\tau) d \tau d t .
\end{aligned}
$$

Letting $\tau=u x$ and $d \tau=u d x$ in Equation (23), we obtain

$$
\begin{aligned}
R[f(t) * g(t)] & =s \int_{0}^{\infty} \exp (-s t) \int_{0}^{t} f(u x) g(u t-u x) d(u x) d t \\
& =s \int_{0}^{\infty} \exp (-s t) \int_{0}^{t} f(u x) g(u(t-x)) u d x d t
\end{aligned}
$$

Letting $y=t-x$ and $d y=d t$ in Equation (24), we obtain

$$
\begin{aligned}
R[f(t) * g(t)] & =s \iint_{0}^{\infty} 0^{\infty} \exp (-s(x+y)) f(u x) g(u y) u d x d y \\
& =s u \iint_{0}^{\infty} \exp (-s(x+y)) f(u x) g(u y) d x d y \\
& =s u \int_{0}^{\infty} \exp (-s x) f(u x) d x \int_{0}^{\infty} \exp (-s y) g(u y) d y \\
& =\frac{u}{s} s \int_{0}^{\infty} \exp (-s x) f(u x) d x s \int_{0}^{\infty} \exp (-s y) g(u y) d y \\
& =\frac{u}{s} F(s, u) G(s, u) .
\end{aligned}
$$

Corollary 1. The Formable transform of $(f * g)^{\prime}$ is given by

$$
B\left[(f * g)^{\prime}\right]=F(s, u) G(s, u)
$$

Proof of Corollary 1. Applying the facts in properties (3) and (4), we obtain

$$
B\left[(f * g)^{\prime}\right]=\frac{s}{u} R(f(t) * g(t))-\frac{s}{u}(f * g)(0) .
$$


But $(f * g)(0)=0$, and hence

$$
\begin{aligned}
B\left[(f * g)^{\prime}\right] & =\frac{s}{u} \frac{u}{s} F(s, u) G(s, u) \\
& =F(s, u) G(s, u) .
\end{aligned}
$$

Here, if we put $g(t)=f(t)$ in Equation (25) we have

$$
B\left[(f * f)^{\prime}\right]=F^{2}(s, u) .
$$

Property 5 (shifting on s-domain). If the function $g(t)$ in a set $W$ is multiplied with the shift function $t^{n}$, then

$$
R\left[t^{n} g(t)\right]=(-u)^{n} s \frac{\partial^{n}}{\partial s^{n}}\left[\frac{R[g(t)]}{s}\right]
$$

Proof of Property 5. We show that Equation (27) is true for $n=1$.

Putting $n=1$ in Equation (27), we have

$$
\begin{aligned}
R[\operatorname{tg}(t)] & =-u s \frac{\partial}{\partial s}\left[\frac{R[g(t)]}{s}\right] \\
& =\frac{-u}{s}\left[s \frac{\partial R[g(t)]}{\partial s}-R[g(t)]\right] \\
& =-u \frac{\partial R[g(t)]}{\partial s}+\frac{u}{s} R[g(t)]
\end{aligned}
$$

Equation (28) becomes

$$
\frac{\partial R[g(t)]}{\partial s}=\frac{-1}{u} R[\operatorname{tg}(t)]+\frac{1}{s} R[g(t)]
$$

If we prove Equation (29), we are finished. We start with the left-hand side of Equation (29) and using the Leibniz rule we obtain

$$
\begin{aligned}
\frac{\partial R[g(t)]}{\partial s} & =\frac{\partial}{\partial s}\left[\frac{s}{u} \int_{0}^{\infty} \exp \left(\frac{-s t}{u}\right) g(t) d t\right] \\
& =\frac{s}{u} \int_{0}^{\infty} \frac{\partial}{\partial s} \exp \left(\frac{-s t}{u}\right) g(t) d t+\frac{1}{u} \int_{0}^{\infty} \exp \left(\frac{-s t}{u}\right) g(t) d t \\
& \left.=\frac{s}{u} \int_{0}^{\infty} \frac{-t}{u} \exp \left(\frac{-s t}{u}\right) g(t) d t\right]+\frac{1}{u} \int_{0}^{\infty} \exp \left(\frac{-s t}{u}\right) g(t) d t \\
& \left.=\frac{-1}{u} \frac{s}{u} \int_{0}^{\infty} t \exp \left(\frac{-s t}{u}\right) g(t) d t\right]+\frac{1}{u} \int_{0}^{\infty} \exp \left(\frac{-s t}{u}\right) g(t) d t \\
& =\frac{-1}{u} R[\operatorname{tg}(t)]+\frac{1}{s} R[g(t)] .
\end{aligned}
$$

The proof is complete for $n=1$.

Assume that Equation (27) is true for $n$ such that

$$
R\left[t^{n} g(t)\right]=(-u)^{n} s \frac{\partial^{n}}{\partial s^{n}}\left[\frac{R[g(t)]}{s}\right] .
$$

We show that

$$
R\left[t^{n+1} g(t)\right]=(-u)^{n+1} s \frac{\partial^{n+1}}{\partial s^{n+1}}\left[\frac{R[g(t)]}{s}\right]
$$


Using the facts in Equations (28) and (30), we have

$$
\begin{gathered}
R\left[t^{n+1} g(t)\right]=R\left[t t^{n} g(t)\right] \\
=-u s \frac{\partial}{\partial s}\left[\frac{R\left[t^{n} g(t)\right]}{s}\right] \\
=-u s \frac{\partial}{\partial s}\left[\frac{(-u)^{n} s \frac{\partial^{n}}{\partial s^{n}}\left[\frac{R[g(t)]}{s}\right]}{s}\right] \\
=-u s\left[\frac{s\left[(-u)^{n} s \cdot \frac{\partial^{n+1}}{\partial s^{n+1}}\left[\frac{R[g(t)]}{s}\right]+(-u)^{n} \frac{\partial^{n}}{\partial s^{n}}\left[\frac{R[g(t)]]}{s}\right]\right]-(-u)^{n} \frac{\partial^{n}}{\partial s^{n}}\left[\frac{R[g(t)]}{s}\right]}{s^{2}}\right] \\
=(-u)^{n+1} s \frac{\partial^{n+1}}{\partial s^{n+1}}\left[\frac{R[g(t)]]}{s}\right] .
\end{gathered}
$$

Remark 1. If the function $g(t)$ has the numerical expansion

$$
g(t)=\sum_{n=0}^{\infty} a_{n} t^{n}
$$

then the Formable transform (see Table A1 in Appendix A) of $\operatorname{tg}(t)$ is given by

$$
\begin{aligned}
R[\operatorname{tg}(t)] & =\sum_{n=0}^{\infty} \frac{(n+1) ! a_{n} u^{n+1}}{s^{n+1}} \\
& =\frac{u}{s} \sum_{n=0}^{\infty} \frac{(n+1) ! a_{n} u^{n}}{s^{n}} \\
& =\frac{u}{s} \sum_{n=0}^{\infty} \frac{\partial}{\partial u} \frac{n ! a_{n} u^{n+1}}{s^{n}} \\
& =\frac{u}{s} \frac{\partial}{\partial u} u \sum_{n=0}^{\infty} \frac{n ! a_{n} u^{n}}{s^{n}} \\
& =\frac{u}{s} \frac{\partial}{\partial u}[u B(s, u)] .
\end{aligned}
$$

The generalization of the previous remark under the condition on $g(t)$ gives us an equivalent form of property (5) as follows:

$$
R\left[t^{n} g(t)\right]=\frac{u^{n}}{s^{n}} \frac{\partial^{n}}{\partial u^{n}}\left(u^{n} B(s, u)\right)
$$

Remark 2. If $g^{(n)}(t)$ is the $n$-th derivative of the function $g(t)$ that is multiplied with the shift function $t^{n}$, then

$$
R\left[t^{n} g^{(n)}(t)\right]=u^{n} \frac{\partial^{n}}{\partial u^{n}}[B(s, u)]
$$

Proof of Remark 2. Consider the right-hand side of Equation (32). Using the Leibniz rule, we obtain

$$
\begin{aligned}
u^{n} \frac{\partial^{n}}{\partial u^{n}}[B(s, u)] & =u^{n} \frac{\partial^{n}}{\partial u^{n}} s \int_{0}^{\infty} \exp (-s t) g(u t) d t \\
& =u^{n} s \int_{0}^{\infty} \exp (-s t) \frac{\partial^{n}}{\partial u^{n}} g(u t) d t \\
& =u^{n} s \int_{0}^{\infty} \exp (-s t) t^{n} g(n)(u t) d t \\
& =s \int_{0}^{\infty} \exp (-s t)(u t)^{n} g^{(n)}(u t) d t \\
& =R\left[t^{n} g^{(n)}(t)\right] .
\end{aligned}
$$

Property 6. If the function $g(t)$ in a set $W$ is divided by the multiple shift function $t^{n}$, then

$$
R\left[\frac{g(t)}{t^{n}}\right]=\frac{s}{u^{n}} \int_{s}^{\infty} \cdots \int_{s}^{\infty} \frac{B(s, u)}{s}(d s)^{n}
$$


Proof of Property 6. Starting with right-hand side of Equation (33), we obtain

$$
\begin{aligned}
\frac{s}{u^{n}} \int_{s}^{\infty} \cdots \int_{s}^{\infty} \frac{B(s, u)}{s}(d s)^{n} & =\frac{s}{u^{n}} \int_{s}^{\infty} \cdots \int_{s}^{\infty} \int_{0}^{\infty} \exp (-s t) g(u t) d t(d s)^{n} \\
& =\frac{s}{u^{n}} \int_{0}^{\infty} g(u t) \int_{s}^{\infty} \cdots \int_{s}^{\infty} \exp (-s t)(d s)^{n} d t \\
& =\frac{s}{u^{n}} \int_{0}^{\infty} \frac{g(u t)}{t^{n}} \exp (-s t) d t \\
& =s \int_{0}^{\infty} \frac{g(u t)}{(u t)^{n}} \exp (-s t) d t \\
& =R\left[\frac{g(t)}{t^{n}}\right] .
\end{aligned}
$$

Property 7. Let the function $g(t)$ be multiplied with the weight function $\exp (\alpha t)$, then

$$
R[\exp (\alpha t) g(t)]=\frac{s}{s-\alpha u} B\left[s, \frac{u}{s-\alpha u}\right]
$$

Proof of Property 7.

$$
\begin{aligned}
R[\exp (\alpha t) g(t)] & =s \int_{0}^{\infty} \exp (-s t) \exp (\alpha u t) g(u t) d t \\
& =s \int_{0}^{\infty} \exp (-(s-\alpha u) t) g(u t) d t
\end{aligned}
$$

Letting $(s-\alpha u) t=s w$, and $d t=\frac{s}{s-\alpha u} d w$ in Equation (36), we have

$$
\begin{aligned}
& =s \int_{0}^{\infty} \exp (-s w) g\left(\frac{u s w}{s-\alpha u}\right) \frac{s}{s-\alpha u} d w \\
& =\frac{s}{s-\alpha u} s \int_{0}^{\infty} \exp (-s w) g\left(\frac{u s w}{s-\alpha u}\right) d w \\
& =\frac{s}{s-\alpha u} B\left[s, \frac{u s}{s-\alpha u}\right] .
\end{aligned}
$$

\section{Duality with Transforms and Some Examples}

In this section, we illustrate the relation between the new transform and other wellknown transforms. Also we compute the Formable transform for some functions to show its applicability and simplicity during the computations.

\subsection{Dualities between Formable Transform and Other Integral Transforms}

- Formable-Laplace duality: let $B(s, u)$ be the Formable transform and $F(s)$ be the Laplace transform of the same function $g(t)$, then it is clear that

$$
B(s, 1)=s F(s) \text {. }
$$

- $\quad$ Formable-Elzaki duality: let $E(u)$ be the Elzaki transform of $g(t)$, then

$$
B(1, u)=\frac{1}{u^{2}} E(u)
$$

- Formable-Sumudu duality: let $G(u)$ be the Sumudu transform of $g(t)$, then

$$
\begin{gathered}
B(1, u)=G(u) \\
B(s, u)=\frac{s}{u} \int_{0}^{\infty} \exp \left(\frac{-s t}{u}\right) g(t) d t . B(1, u)=\frac{1}{u} \int_{0}^{\infty} \operatorname{ext}\left(\frac{-t}{u}\right) g(t) d t=G(u) .
\end{gathered}
$$

- Formable-Natural duality: let $R(s, u)$ be the Natural transform of $g(t)$, then

$$
B(s, u)=s R(s, u) .
$$


- Formable-Shehu duality: let $\mathrm{V}(\mathrm{s}, \mathrm{u})$ be the Shehu transform of $g(t)$, then,

$$
B(s, u)=\frac{s}{u} V(s, u) .
$$

- Formable-ARA duality: let $\mathcal{G}_{n}(\mathrm{~s})$ be the ARA transform of $g(t)$, then

$$
B(s, 1)=\mathcal{G}_{1}(s) .
$$

Furthermore, substituting $u=1$ in $R\left[t^{n-1} g(t)\right]$, we obtain

$$
R\left[t^{n-1} g(t)\right]=\mathcal{G}_{n}[g(t)](\mathrm{s})
$$

\subsection{Examples of Formable Transform for Some Functions}

In the following arguments, we compute the Formable transform for some functions to demonstrate its simplicity and applicability through computations.

Example 1. Let the function $g(t)=1$,

Then

$$
R[g(t)]=1,
$$

\section{Proof of Example 1.}

$$
R[1]=\frac{s}{u} \int_{0}^{\infty} \exp \left(\frac{-s t}{u}\right) d t=\frac{s}{u_{\alpha \rightarrow \infty}} \lim _{\alpha \rightarrow}\left[\frac{-u}{s} \exp \left(\frac{-s t}{u}\right)\right]_{t=0}^{\alpha}=1 .
$$

Example 2. Let the function $g(t)=t$, then

$$
R[g(t)]=\frac{u}{s} .
$$

Proof of Example 2.

$$
\begin{aligned}
R[\mathrm{t}] & =\frac{s}{u} \int_{0}^{\infty} \exp \left(\frac{-s t}{u}\right) d t \\
& =\frac{s}{u} \lim _{\alpha \rightarrow \infty}\left[\frac{-u}{s} t \exp \left(\frac{-s t}{u}\right)-\frac{u^{2}}{s^{2}} \exp \left(\frac{-s t}{u}\right)\right]_{t=0}^{\alpha}=\frac{u}{s} .
\end{aligned}
$$

Example 3. Let the function $(t)=\frac{t^{2}}{2}$, then

$$
R[g(t)]=\frac{u^{2}}{s^{2}} .
$$

\section{Proof of Example 3.}

$$
\begin{aligned}
R\left[\frac{t^{2}}{2}\right] & =\frac{s}{u} \int_{0}^{\infty} \exp \left(\frac{-s t}{u}\right) \frac{t^{2}}{2} d t \\
& =\frac{s}{2 u} \lim _{\alpha \rightarrow \infty}\left[\frac{-u}{s} t^{2} \exp \left(\frac{-s t}{u}\right)-2 \frac{u^{2}}{s^{2}} \mathrm{t} \exp \left(\frac{-s t}{u}\right)-2 \frac{u^{3}}{s^{3}} \exp \left(\frac{-s t}{u}\right)\right]_{t=0}^{\alpha} \\
& =\frac{u^{2}}{s^{2}} .
\end{aligned}
$$


Example 4. Let the function $g(t)=\frac{t^{n}}{n !}$, then

$$
R[g(t)]=\frac{u^{n}}{s^{n}} .
$$

\section{Proof of Example 4.}

$$
\begin{aligned}
R\left[\frac{t^{n}}{n !}\right] & =\frac{s}{u} \int_{0}^{\infty} \exp \left(\frac{-s t}{u}\right) \frac{t^{n}}{n !} d t \\
& =\frac{s}{u n !} \int_{0}^{\infty} \exp \left(\frac{-s t}{u}\right) t^{n} d t \\
& +=\frac{1}{(n-1) !} \int_{0}^{\infty} \exp \left(\frac{-s t}{u}\right) t^{n-1} d t \\
& \vdots \\
& =\frac{u^{n-2}}{s^{n-2}} \int_{0}^{\infty} \exp \left(\frac{-s t}{u}\right) t d t=\frac{u^{n}}{s^{n}} .
\end{aligned}
$$

Example 5. Let the function $g(t)=\exp (\alpha t)$, then

$$
R[g(t)]=\frac{s}{s-\alpha u} .
$$

Proof of Example 5.

$$
\begin{aligned}
R[\exp (\alpha t)] & =\frac{s}{u} \int_{0}^{\infty} \exp \left(\frac{-s t}{u}\right) \exp (\alpha t) d t \\
& =\frac{s}{u} \int_{0}^{\infty} \exp \left(\frac{(\alpha u-s) t}{u}\right) d t \\
& =\frac{s}{u} \lim _{\beta \rightarrow \infty}\left[\frac{u}{\alpha u-s} \exp \left(\frac{(\alpha u-s) t}{u}\right)\right]_{t=0}^{\beta}=\frac{s}{s-\alpha u} .
\end{aligned}
$$

Example 6. Let the function $g(t)=t \exp (\alpha t)$, then

$$
R[g(t)]=\frac{s u}{(s-\alpha u)^{2}} .
$$

\section{Proof of Example 6.}

$$
\begin{aligned}
R\left[t^{n} \exp (\alpha t)\right] & =\frac{s}{u} \int_{0}^{\infty} \exp \left(\frac{-s t}{u}\right) \operatorname{texp}(\alpha t) d t \\
& =\frac{s}{u} \int_{0}^{\infty} \mathrm{t} \exp \left(-\frac{(s-\alpha u) t}{u}\right) d t \\
& =\frac{s}{u} \lim _{\beta \rightarrow \infty}\left[\frac{-u}{s-\alpha u} t \exp \left(-\frac{(s-\alpha u) t}{u}\right)-\frac{u^{2}}{(s-\alpha u)^{2}} \exp \left(-\frac{(s-\alpha u) t}{u}\right)\right]_{t=0}^{\beta} \\
& =\frac{s u}{(s-\alpha u)^{2}} .
\end{aligned}
$$

Example 7. Let the function $g(t)=\frac{t^{n}}{n !} \exp (\alpha t)$, then

$$
R[g(t)]=\frac{s u^{n}}{(s-\alpha u)^{n+1}} .
$$


Proof of Example 7.

$$
\begin{aligned}
R\left[t^{n} \exp (\alpha t)\right] & =\frac{s}{u} \int_{0}^{\infty} t^{n} \exp \left(-\frac{(s-\alpha u) t}{u}\right) d t \\
& =\frac{s n}{s-\alpha u} \int_{0}^{\infty} t^{n-1} \exp \left(-\frac{(s-\alpha u) t}{u}\right) d t \\
& =\frac{s u n(n-1)}{(s-\alpha u)^{2}} \int_{0}^{\infty} t^{n-2} \exp \left(-\frac{(s-\alpha u) t}{u}\right) d t \\
& \vdots \\
& =\frac{s u^{n} n !}{(s-\alpha u)^{n+1}} .
\end{aligned}
$$

Example 8. Let the function $g(t)=\sin (\alpha t)$, then

$$
R[g(t)]=\frac{\alpha s u}{s^{2}+\alpha^{2} u^{2}} .
$$

\section{Proof of Example 8.}

$$
\begin{aligned}
R[\sin (\alpha t)] & =\frac{s}{u} \int_{0}^{\infty} \exp \left(\frac{-s t}{u}\right) \sin (\alpha t) d t \\
& =\frac{s}{u} \lim _{\beta \rightarrow \infty}\left[\exp \left(\frac{-s t}{u}\right) \frac{\frac{-s}{u} \sin (\alpha t)-\alpha \cos (\alpha t)}{\frac{s^{2}}{u^{2}}+\alpha^{2}}\right]_{t=0}^{\beta} \\
& =\frac{s}{u}\left[\frac{\alpha}{\frac{s^{2}}{u^{2}}+\alpha^{2}}\right] \\
& =\frac{\alpha s u}{s^{2}+\alpha^{2} u^{2}}
\end{aligned}
$$

Example 9. Let the function $g(t)=\cos (\alpha t)$, then

$$
R[g(t)]=\frac{s^{2}}{s^{2}+\alpha^{2} u^{2}} .
$$

\section{Proof of Example 9.}

$$
\begin{aligned}
R[\cos (\alpha t)] & =\frac{s}{u} \int_{0}^{\infty} \exp \left(\frac{-s t}{u}\right) \cos (\alpha t) d t \\
& =\frac{s}{u} \lim \left[\exp \left(\frac{-s t}{u}\right) \frac{\frac{-s}{u} \cos (\alpha t)+\alpha \sin (\alpha t)}{\frac{s^{2}}{u^{2}}+\alpha^{2}}\right]_{t=0}^{\beta} \\
& =\frac{s}{u}\left[\frac{\frac{s}{u}}{\frac{s^{2}}{u^{2}}+\alpha^{2}}\right] \\
& =\frac{s^{2}}{s^{2}+\alpha^{2} u^{2}} .
\end{aligned}
$$

Example 10. Let the function $g(t)=\frac{\sinh (\alpha t)}{\alpha}$, then

$$
R[g(t)]=\frac{s u}{s^{2}-\alpha^{2} u^{2}} .
$$


Proof of Example 10.

$$
\begin{aligned}
R[\sinh (\alpha t)]= & \frac{s}{u} \int_{0}^{\infty} \exp \left(\frac{-s t}{u}\right) \sinh (\alpha t) d t \\
& =\frac{s}{u} \lim _{\beta \rightarrow \infty}\left[\exp \left(\frac{-s t}{u}\right) \frac{\frac{-s}{u} \sinh (\alpha t)-\alpha \cosh (\alpha t)}{\frac{s^{2}}{u^{2}}-\alpha^{2}}\right]_{t=0}^{\beta} \\
& =\frac{s}{u}\left[\frac{\alpha}{\frac{s^{2}}{u^{2}}-\alpha^{2}}\right] \\
& =\frac{\alpha s u}{s^{2}-\alpha^{2} u^{2}} .
\end{aligned}
$$

Example 11. Let the function $g(t)=\cosh (\alpha t)$, then

$$
R[g(t)]=\frac{s^{2}}{s^{2}-\alpha^{2} u^{2}}
$$

\section{Proof of Example 11.}

$$
\begin{aligned}
R[\cosh (\alpha t)] & =\frac{s}{u} \int_{0}^{\infty} \exp \left(\frac{-s t}{u}\right) \cosh (\alpha t) d t \\
& =\frac{s}{u} \lim \left[\exp \left(\frac{-s t}{u}\right) \frac{\frac{-s}{u} \cosh (\alpha t)-\alpha \sinh (\alpha t)}{\frac{s^{2}}{u^{2}}-\alpha^{2}}\right]_{t=0}^{\beta} \\
& =\frac{s}{u}\left[\frac{\frac{s}{u}}{\frac{s^{2}}{u^{2}}-\alpha^{2}}\right] \\
& =\frac{s^{2}}{s^{2}-\alpha^{2} u^{2}} .
\end{aligned}
$$

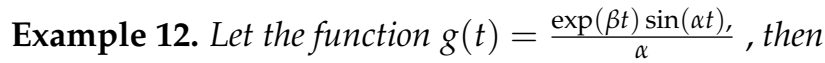

$$
R[g(t)]=\frac{s u}{(s-\beta u)^{2}+\alpha^{2} u^{2}} .
$$

\section{Proof of Example 12.}

$$
\begin{aligned}
& R[\exp (\beta t) \sin (\alpha t)]=\frac{s}{u} \int_{0}^{\infty} \exp \left(\frac{-s t}{u}\right) \exp (\beta t) \sin (\alpha t) d t \\
& =\frac{s}{u} \int_{0}^{\infty} \exp \left(-\frac{(s-\beta u)}{u} t\right) \sin (\alpha t) d t \\
& =\frac{s}{u}\left[\lim _{\gamma \rightarrow \infty}\left[\frac{-u}{s-\beta u} \exp \left(-\frac{(s-\beta u)}{u} t\right) \sin (\alpha t)\right]_{t=0}^{\gamma}+\right. \\
& \left.\frac{\alpha u}{s-\beta u} \int_{0}^{\infty} \exp \left(-\frac{(s-\beta u)}{u} t\right) \cos (\alpha t) d t\right] \\
& =\frac{\alpha s}{s-\beta u} \int_{0}^{\infty} \exp \left(-\frac{(s-\beta u)}{u} t\right) \cos (\alpha t) d t \\
& =\frac{\alpha s}{s-\beta u}\left[\lim _{\gamma \rightarrow \infty}\left[\frac{-u}{s-\beta u} \exp \left(-\frac{(s-\beta u)}{u} t\right) \cos (\alpha t)\right]_{t=0}^{\gamma}-\right. \\
& \left.\frac{\alpha u}{s-\beta u} \int_{0}^{\infty} \exp \left(-\frac{(s-\beta u)}{u} t\right) \sin (\alpha t) d t\right] \\
& =\frac{\alpha s}{s-\beta u}\left[\frac{u}{s-\beta u}\right. \\
& \left.-\frac{\alpha u}{s-\beta u} \int_{0}^{\infty} \exp \left(-\frac{(s-\beta u)}{u} t\right) \sin (\alpha t) d t\right] .
\end{aligned}
$$

Simplifying the required integral, we obtain:

$$
R[\exp (\beta t) \sin (\alpha t)] \frac{\alpha s u}{(s-\beta u)^{2}+\alpha^{2} u^{2}} .
$$


Example 13. Let the function $(t)=\exp (\beta t) \cos (\alpha t)$, then

$$
R[g(t)]=\frac{s(s-\beta u)}{(s-\beta u)^{2}+\alpha^{2} u^{2}} .
$$

Proof of Example 13. By similar computations to Example 12, we obtain the result.

\section{Applications}

In this section, we introduce some applications using the Formable transform in solving ordinary and partial differential equations using several properties of the new transform, such as the derivative property, the convolution property and the shifting theorem of the Formable transform.

Example 14. Consider the first order differential equation

$$
y^{\prime}(t)+5 y(t)=0
$$

subject to the initial condition

$$
y(0)=2 \text {. }
$$

Solution. Applying the Formable transform on both sides of Equation (57).

$$
R\left[y^{\prime}(t)\right]+R[5 y(t)]=R[0]
$$

we obtain

$$
\frac{s}{u} B(s, u)-\frac{s}{u} y(0)+5 B(s, u)=0 .
$$

Substituting the initial condition of (58) and simplifying Equation (59), we have

$$
\left[\frac{s}{u}+5\right] B(s, u)=2 \frac{s}{u} \cdot B(s, u)=\frac{2 s}{s+5 u} .
$$

Taking the inverse Formable transform of Equation (60), we obtain the solution

$$
y(t)=2 \exp (-5 t)
$$

Example 15. Consider the second order differential equation

$$
y^{\prime \prime}(t)+2 y^{\prime}(t)+5 y(t)=\exp (-t) \sin (t)
$$

subject to the initial conditions

$$
y^{\prime}(0)=1, y(0)=0
$$

Solution. Applying the Formable transform in Equation (62) and using property (3) and the result in Equation (55), we obtain

$$
\begin{gathered}
R\left[y^{\prime \prime}(t)\right]+R\left[2 y^{\prime}(t)\right]+R[5 y(t)]=R[\exp (-t) \sin (t)], \\
\frac{s^{2}}{u^{2}} B(s, u)-\frac{s^{2}}{u^{2}} y(0)-\frac{s}{u} y^{\prime}(0)+2 \frac{s}{u} B(s, u)-2 \frac{s}{u} y(0)+5 B(s, u)=\frac{s u}{(s+u)^{2}+u^{2}}
\end{gathered}
$$

Substituting the initial conditions of (63) and simplifying Equation (64), we obtain

$$
\left[\frac{s^{2}+2 s u+5 u^{2}}{u^{2}}\right] B(s, u)=\frac{s u}{(s+u)^{2}+u^{2}}+\frac{s}{u} .
$$


Hence,

$$
B(s, u)=\frac{s u^{3}}{\left[(s+u)^{2}+4 u^{2}\right]\left[(s+u)^{2}+u^{2}\right]}+\frac{s u}{\left[(s+u)^{2}+4 u^{2}\right]}
$$

Simplifying Equation (65),

$$
B(s, u)=\frac{1}{3} \frac{s u}{\left[(s+u)^{2}+u^{2}\right]}+\frac{2}{3} \frac{s u}{\left[(s+u)^{2}+4 u^{2}\right]} .
$$

Taking the inverse Formable transform of Equation (66), we obtain

$$
y(t)=\frac{1}{3} \exp (-t) \sin (t)+\frac{2}{3} \exp (-t) \sin (2 t)
$$

Example 16. Consider the second order differential equation

$$
y^{\prime \prime}(t)-3 y^{\prime}(t)+2 y(t)=\exp (3 t)
$$

subject to the initial conditions

$$
y^{\prime}(0)=0, y(0)=1
$$

Solution. Applying the Formable transform on both sides of Equation (68) and using the result in Equation (47), we have

$$
\begin{gathered}
R\left[y^{\prime \prime}(t)\right]-R\left[3 y^{\prime}(t)\right]+R[2 y(t)]=R[\exp (3 t)] . \\
\frac{s^{2}}{u^{2}} B(s, u)-\frac{s^{2}}{u^{2}} y(0)-\frac{s}{u} y^{\prime}(0)-3 \frac{s}{u} B(s, u)+3 \frac{s}{u} y(0)+2 B(s, u)=\frac{s}{s-3 u},
\end{gathered}
$$

Substituting the initial conditions of (69) and simplifying Equation (70), we obtain

$$
B(s, u)=\frac{s u^{2}}{(s-u)(s-2 u)(s-3 u)}+\frac{s^{2}-3 s u}{(s-u)(s-2 u)} .
$$

After simplifying Equation (71) and taking the inverse Formable transform, we have

$$
y(t)=\frac{5}{2} \exp (t)-2 \exp (2 t)+\frac{1}{2} \exp (3 t)
$$

Example 17. Consider the Bessel differential equation (with polynomial coefficients)

$$
t y^{\prime \prime}(t)+y^{\prime}(t)+t y(t)=0,
$$

with the initial conditions.

$$
y(0)=1, y^{\prime}(0)=1
$$

Solution. Applying, the Formable transform on both sides of Equation (73), we obtain

$$
R\left[t y^{\prime \prime}(t)\right]+R\left[y^{\prime}(t)\right]+R[t y(t)]=R[0]
$$

Using the facts in Equations(18) and (27) in Equation (75), we obtain

$$
\begin{aligned}
& -u s \frac{\partial}{\partial s}\left[\frac{\frac{s^{2}}{u^{2}} B(s, u)-\frac{s^{2}}{u^{2}} y(0)-\frac{s}{u} y^{\prime}(0)}{s}\right]+\frac{s}{u} B(s, u)-\frac{s}{u}-u s \frac{\partial}{\partial s}\left[\frac{B(s, u)}{s}\right]=0 \\
& -u s \frac{\partial}{\partial s}\left[\frac{s}{u^{2}} B(s, u)-\frac{s}{u^{2}} y(0)-\frac{1}{u} y^{\prime}(0)\right]+\frac{s}{u} B(s, u)-\frac{s}{u}-u s \frac{\partial}{\partial s}\left[\frac{B(s, u)}{s}\right]=0
\end{aligned}
$$


Substituting the initial conditions, we obtain

$$
-u s \frac{\partial}{\partial s}\left[\frac{s}{u^{2}} B(s, u)-\frac{s}{u^{2}}-\frac{1}{u}\right]+\frac{s}{u} B(s, u)-\frac{s}{u}-u s \frac{\partial}{\partial s}\left[\frac{B(s, u)}{s}\right]=0 .
$$

After simple computations, Equation (76) becomes

$$
\frac{\partial B(s, u)}{B(s, u)}=\frac{u^{2}}{s\left(s^{2}+u^{2}\right)} d s .
$$

Integrating both sides of Equation (77), we obtain

$$
\ln B(s, u)=\ln s-\frac{1}{2} \log \left(s^{2}+u^{2}\right)+\ln (c), B(s, u)=\frac{c s}{\sqrt{s^{2}+u^{2}}} .
$$

Taking the inverse Formable transform of Equation (78) and letting $c=1$, we obtain

$$
y(t)=J_{0}(t) .
$$

Example 18. Consider the nonhomogeneous partial differential equation

$$
u_{t t}=u_{x x}+\sin \pi x
$$

with the initial boundary conditions

$$
\left\{\begin{array}{c}
u(0, t)=u(1, t)=0 \\
u(x, 0)=u_{t}(x, 0)=0
\end{array}\right\}
$$

Solution. Applying the Formable transform on both sides of Equation (80) and using the facts in Equations (18) and (20), we obtain

$$
\frac{s^{2}}{u^{2}} B(x, s, u)-\frac{s^{2}}{u^{2}} u(x, 0)-\frac{s}{u} u_{t}(x, 0)=\frac{\partial^{2}}{\partial x^{2}} B(x, s, u)+\sin \pi x
$$

Substituting the initial conditions of (81) in Equation (82), we have

$$
\frac{\partial^{2}}{\partial x^{2}} B(x, s, u)-\frac{s^{2}}{u^{2}} B(x, s, u)=-\sin \pi x .
$$

The general solution of the differential Equation (83) can be written as

$$
B(x, s, u)=B_{h}(x, s, u)+B_{p}(x, s, u),
$$

where $B_{h}(x, s, u)=C_{1} \exp \left(\frac{s}{u} x\right)+C_{2} \exp \left(-\frac{s}{u} x\right)$ is the homogeneous part of the general solution of Equation (83) and $B_{p}(x, s, u)=A \sin \pi x+B \cos \pi x$ is the nonhomogeneous part of the general solution of Equation (83).

To find $A$ and $B$ in $B_{p}(x, s, u)$, we substitute $B_{p}(x, s, u)$ in Equation (83) to give

$$
B_{p}(x, s, u)=\frac{u^{2}}{s^{2}+\pi^{2} u^{2}} \sin \pi x,
$$

since

$$
A=\frac{u^{2}}{s^{2}+\pi^{2} u^{2}}, \text { and } B=0 .
$$

Hence, Equation (84) becomes

$$
B(x, s, u)=C_{1} \exp \left(\frac{s}{u} x\right)+C_{2} \exp \left(-\frac{s}{u} x\right)+\frac{u^{2}}{s^{2}+\pi^{2} u^{2}} \sin \pi x .
$$


Substituting the boundary conditions of (81) in Equation (85), we obtain $C_{1}=C_{2}=0$, and therefore

$$
B(x, s, u)=\frac{u^{2}}{s^{2}+\pi^{2} u^{2}} \sin \pi x \cdot B(x, s, u)=\frac{u}{s} \frac{u}{s} \frac{s^{2}}{s^{2}+\pi^{2} u^{2}} \sin \pi x
$$

In Equation (86), we consider

$$
F(s, u)=\frac{u}{s} \rightarrow f(t)=t, \text { and } G(s, u)=\frac{s^{2}}{s^{2}+\pi^{2} u^{2}} \rightarrow g(t)=\cos \pi t .
$$

Hence, taking the inverse Formable transform of both sides of Equation (86), and using the convolution property, we obtain

$$
\begin{aligned}
u(x, t) & =(f(t) * g(t)) \sin \pi x . \\
& =\sin \pi x \int_{0}^{t} \tau \cos \pi(t-\tau) d \tau \\
& =\frac{\sin \pi x}{\pi^{2}}[1-\cos \pi t] .
\end{aligned}
$$

Hence, the solution of Equation (80) with the conditions of (81) is

$$
u(x, t)=\frac{\sin \pi x}{\pi^{2}}[1-\cos \pi t]
$$

\section{Conclusions}

In this article, we presented a new integral transform called the Formable transform. We introduced the sufficient conditions for the existence of the new transform. The duality with other transforms was explained, and some essential properties were proved. The applicability and accuracy of the new transform were shown by solving examples for both ordinary and partial differential equations. In addition, we presented tables in the Appendix A to compare the Formable transform with other well-known transforms and to illustrate the simplicity and ability of the new transform through applications. In the future, we intend to solve fractional differential equations and integral equations using the Formable transform. Furthermore, we plan to combine the transform with other analytical methods to solve nonlinear problems such as Duffing oscillator and MEMS oscillator problems and some fractional differential equations in the conformable sense.

Author Contributions: Conceptualization, R.Z.S. and B.f.G.; methodology R.Z.S. and B.f.G.; validation, R.Z.S. and B.f.G.; formal analysis, R.Z.S.; writing-original draft preparation, R.Z.S. and B.f.G.; writing-review and editing, R.Z.S.; supervision, R.Z.S.; project administration, R.Z.S. and B.f.G. All authors have read and agreed to the published version of the manuscript.

Funding: This research received no external funding.

Data Availability Statement: There is no data needed.

Conflicts of Interest: The authors declare no conflict of interest.

\section{Appendix A}

Table A1. Formable transform of some special functions.

\begin{tabular}{ccc}
\hline No. & $\boldsymbol{g}(\boldsymbol{t})$ & $\boldsymbol{B}(s, \boldsymbol{u})$ \\
\hline 1 & 1 & 1 \\
2 & $t$ & $\frac{u}{s}$ \\
3 & $\frac{t^{n}}{n !} ;$ for $n=0,1,2, \ldots$ & $\frac{u^{n}}{s^{n}}$ \\
4 & $\exp (\alpha t)$ & $\frac{s}{s-\alpha u}$ \\
5 & $\frac{t^{n}}{n !} \exp (\alpha t)$ & $\frac{s u^{n}}{(s-\alpha u)^{n+1}}$ \\
\hline
\end{tabular}


Table A1. Cont.

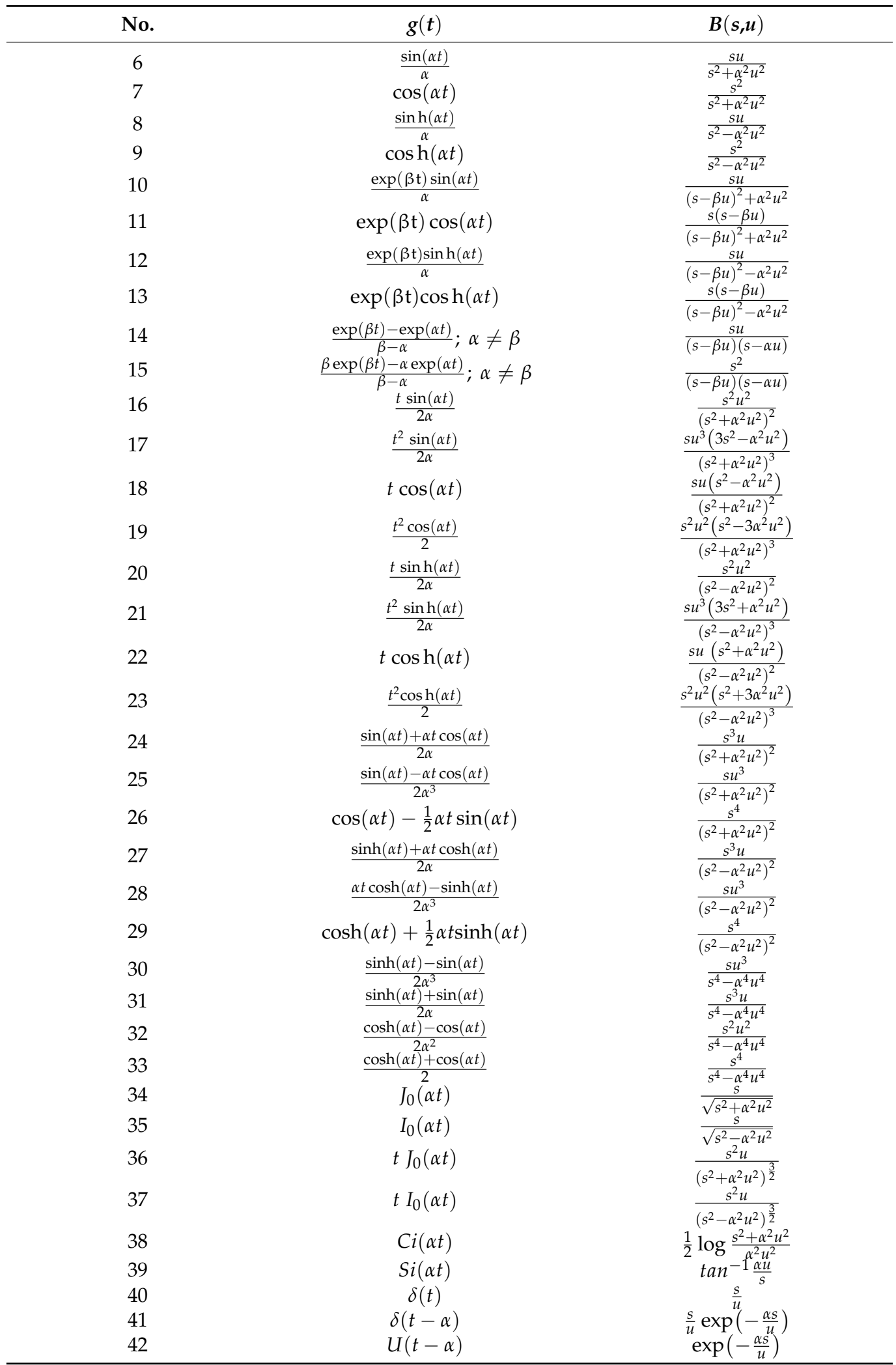


Table A2. General properties of Formable transform.

\begin{tabular}{|c|c|c|}
\hline No. & Property & Definition \\
\hline 1 & Definition & $B(s, u)=\frac{s}{u} \int_{0}^{\infty} \exp \left(\frac{-s t}{u}\right) g(t) d t$ \\
\hline 2 & Inverse & $g(t)=\frac{1}{2 \pi i} \int_{c-i \infty}^{c+i \infty} \frac{1}{s} \exp \left(\frac{s t}{u}\right) B(s, u) d s$ \\
\hline 3 & Derivative & $R\left[g^{n}(t)\right]=\frac{s^{n}}{u^{n}} B(s, u)-\sum_{k=0}^{n-1}\left(\frac{s}{u}\right)^{n-k} g^{(k)}(0)$ \\
\hline 4 & Product shift & $\begin{aligned} R\left[t^{n} g(t)\right] & =\frac{u^{n}}{s^{n}} \frac{\partial^{n}}{\partial u^{n}}\left[u^{n} B(s, u)\right] \\
& =(-u)^{n} s \frac{\partial^{n}}{\partial s^{n}}\left[\frac{R[g(t)]}{s}\right]\end{aligned}$ \\
\hline 5 & Product shift and derivative & $R\left[t^{n} g^{n}(t)\right]=u^{n} \frac{\partial^{n}}{\partial u^{n}}[B(s, u)]$ \\
\hline 6 & Division shift & $R\left[\frac{g(t)}{t^{n}}\right]=\frac{s}{u^{n}} \int_{s}^{\infty} \cdots \int_{s}^{\infty} \frac{B(s, u)}{s}(d s)^{n}$ \\
\hline 7 & Convolution & $B[f * g]=\frac{u}{s} F(s, u) G(s, u)$ \\
\hline
\end{tabular}

Table A3. Important functions and definitions.

\begin{tabular}{ccc}
\hline No. & Function & Definition \\
\hline 1 & Bessel function & $J_{n}(x)=\frac{x^{n}}{2^{n} \Gamma(n+1)} \times\left\{1-\frac{x^{2}}{2(2 n+2)}+\frac{x^{4}}{2.4(2 n+2)(2 n+4)}-\cdots\right\}$ \\
2 & Modified Bessel function & $I_{n}(x)=i^{-n} J_{n}(i x)=$ \\
& & $\frac{x^{n}}{2^{n} \Gamma(n+1)} \times\left\{1+\frac{x^{2}}{2(2 n+2)}+\frac{x^{4}}{2.4(2 n+2)(2 n+4)}-\cdots\right\}$ \\
3 & Sine integral & $\operatorname{Si}(t)=\int_{0}^{t} \frac{\sin u}{u} d u$ \\
4 & Cosine integral & $C i(t)=-\int_{\mathrm{t}}^{\infty} \frac{\cos u}{u} d u$ \\
\hline
\end{tabular}

Table A4. Some integral transforms.

\begin{tabular}{ccc}
\hline No. & Integral Transform & Definition \\
\hline 1 & Laplace transform & $£[g(t)]=G(s)=\int_{0}^{\infty} \exp (-s t) g(t) d t$ \\
2 & Fourier transform & $F[g(t)]=g(w)=\frac{1}{\sqrt{2} \pi} \int_{-\infty}^{\infty} \exp (-i w t) g(t) d t$ \\
3 & Mellin transform & $M[g(s) ; s](s)=g^{*}(s)=\int_{0}^{\infty} x^{s-1} g(x) d x$ \\
4 & Elzaki transform & $E[g(t)](u)=T(u)=u \int_{0}^{\infty} \exp \left(\frac{-t}{u}\right) g(t) d t$ \\
5 & Sumudu transform & $S[g(t)](u)=G(u)=\frac{1}{u} \int_{0}^{\infty} \exp \left(\frac{-t}{u}\right) g(t) d t$ \\
6 & Natural transform & $N^{+}[g(t)](s, u)=R(s, u)=\frac{1}{u} \int_{0}^{\infty} \exp \left(\frac{-s t}{u}\right) g(t) d t, s, u>0$ \\
7 & Shehu transform & $\mathbb{S}[g(t)](s, u)=V(s, u)=\int_{0}^{\infty} \exp \left(\frac{-s t}{u}\right) g(t) d t, s, u>0$ \\
8 & ARA transform & $\mathcal{G}_{n}[g(t)](s)=G(n, s)=s \int_{0}^{\infty} t^{n-1} \exp (-s t) g(t) d t, s>0$ \\
\hline
\end{tabular}


Table A5. Comprehensive list of the Formable transforms $\mathrm{B}(\mathrm{s}, \mathrm{u})$ and their relationship with the Natural transforms $R(s, u)$, the Shehu transforms $V(s, u)$ and the ARA transforms $G(m, s)$.

\begin{tabular}{|c|c|c|c|c|c|}
\hline No. & $g(t)$ & $B(s, u)$ & $V(s, u)$ & $R(s, u)$ & $G(m, s)$ \\
\hline 1 & 1 & 1 & $\frac{u}{s}$ & $\frac{1}{s}$ & $\frac{\Gamma(m)}{c m-1}$ \\
\hline 2 & $t$ & $\frac{u}{s}$ & $\frac{u^{2}}{s^{2}}$ & $\frac{s}{s^{2}}$ & $\frac{\Gamma(m+1)}{s^{m}}$ \\
\hline 3 & $\begin{array}{l}\frac{t^{n}}{n !} ; \text { for } n= \\
0,1,2, \ldots\end{array}$ & $\frac{u^{n}}{s^{n}}$ & $\frac{u^{s^{2}}}{s^{n+1}}$ & $\frac{u^{n}}{s^{n+1}}$ & $\frac{s^{1-n-m} \Gamma(m+n)}{n !}$ \\
\hline 4 & $\exp (\alpha t)$ & $\frac{s}{s-\alpha u}$ & $\frac{u}{s-\alpha u}$ & $\frac{1}{s-\alpha u}$ & $\frac{s \Gamma(m)}{(s-\alpha)^{m}}$ \\
\hline 5 & $\frac{\sin (\alpha t)}{\alpha}$ & $\frac{s u}{s^{2}+\alpha^{2} u^{2}}$ & $\frac{u^{2}}{s^{2}+\alpha^{2} u^{2}}$ & $\frac{u}{s^{2}+\alpha^{2} u^{2}}$ & $\frac{s}{2 \alpha i} \Gamma(m)\left(\frac{1}{(s-i \alpha)^{m}}-\frac{1}{(s+i \alpha)^{m}}\right)$ \\
\hline 6 & $\cos (\alpha t)$ & $\frac{s^{2}}{s^{2}+\alpha^{2} u^{2}}$ & $\frac{s u}{s^{2}+\alpha^{2} u^{2}}$ & $\frac{s}{s^{2}+\alpha^{2} u^{2}}$ & $\frac{s}{2 i} \Gamma(m)\left(\frac{1}{(s-i \alpha)^{m}}+\frac{1}{(s+i \alpha)^{m}}\right)$ \\
\hline 7 & $\frac{\sinh (\alpha t)}{\alpha}$ & $\frac{s u}{s^{2}-\alpha^{2} u^{2}}$ & $\frac{u^{2}}{s^{2}-\alpha^{2} u^{2}}$ & $\frac{u}{s^{2}-\alpha^{2} u^{2}}$ & $\frac{s}{2 \alpha}\left(\frac{-\alpha^{2}}{s}+s\right)^{-m} \Gamma(m)\left[-\left(1-\frac{\alpha}{s}\right)^{m}+\left(\frac{\alpha+s}{s}\right)^{m}\right]$ \\
\hline 8 & $\cosh (\alpha t)$ & $\frac{s^{2}}{s^{2}-\alpha^{2} u^{2}}$ & $\frac{s u}{s^{2}-\alpha^{2} u^{2}}$ & $\frac{s}{s^{2}-\alpha^{2} u^{2}}$ & $\frac{s}{2}\left(s^{2}-\alpha^{2}\right)^{-m} \Gamma(m)\left[(s-|\alpha|)^{m}+(s+|\alpha|)^{m}\right]$ \\
\hline 9 & $\frac{\exp (\beta t) \sin (\alpha t)}{\alpha}$ & $\frac{s u}{(s-\beta u)^{2}+\alpha^{2} u^{2}}$ & $\frac{u^{2}}{(s-\beta u)^{2}+\alpha^{2} u^{2}}$ & $\frac{u}{(s-\beta u)^{2}+\alpha^{2} u^{2}}$ & $\frac{s}{\alpha}(s-\beta)^{-m} \Gamma(m)\left(1+\frac{\alpha^{2}}{(\beta-s)^{2}}\right)^{\frac{-m}{2}} \sin \left(m \tan ^{-1} \frac{\alpha}{s-\beta}\right)$ \\
\hline 10 & $\exp (\beta \mathrm{t}) \cos (\alpha t)$ & $\frac{s(s-\beta u)}{(s-\beta u)^{2}+\alpha^{2} u^{2}}$ & $\frac{u(s-\beta u)}{(s-\beta u)^{2}+\alpha^{2} u^{2}}$ & $\frac{s-\beta u}{(s-\beta u)^{2}+\alpha^{2} u^{2}}$ & $s(s-\beta)^{-m} \Gamma(m)\left(1+\frac{\alpha^{2}}{(\beta-s)^{2}}\right)^{\frac{-m}{2}} \cos \left(m \tan ^{-1} \frac{\alpha}{\beta-s}\right)$ \\
\hline 11 & $\frac{\exp (\beta t) \sinh (\alpha t)}{\alpha}$ & $\frac{s u}{(s-\beta u)^{2}-\alpha^{2} u^{2}}$ & $\frac{u^{2}}{(s-\beta u)^{2}-\alpha^{2} u^{2}}$ & $\frac{u}{(s-\beta u)^{2}-\alpha^{2} u^{2}}$ & $\begin{array}{c}\frac{s}{2 \alpha}(s-\beta)^{-m} \Gamma(m)\left(1-\frac{\alpha^{2}}{(\beta-s)^{2}}\right)^{-m}\left[-\left(1+\frac{\alpha}{\beta-s}\right)^{m}+\right. \\
\left.\left(1+\frac{\alpha}{s-\beta}\right)^{m}\right]\end{array}$ \\
\hline 12 & $\exp (\beta t) \cosh (\alpha t)$ & $\frac{s(s-\beta u)}{(s-\beta u)^{2}-\alpha^{2} u^{2}}$ & $\frac{u(s-\beta u)}{(s-\beta u)^{2}-\alpha^{2} u^{2}}$ & $\frac{s-\beta u}{(s-\beta u)^{2}-\alpha^{2} u^{2}}$ & $\begin{array}{c}\frac{s}{2}\left(\left(s^{2}-\beta^{2}\right)\right)^{-m} \Gamma(m)\left[\left(1+\frac{\sqrt{\alpha^{2}}}{\beta-s}\right)^{-m}+\right. \\
\left.\left(1+\frac{\sqrt{\alpha^{2}}}{s-\beta}\right)^{-m}\right]\end{array}$ \\
\hline
\end{tabular}

\section{References}

1. Kilbas, A.A.; Srivastava, H.M.; Trujillo, J.J. Theory and Applications of Fractional Differential Equations; Elsevier: Amsterdam, The Netherlands, 2006.

2. Roubíček, T. Nonlinear Partial Differential Equations with Applications; Springer Science \& Business Media: Berlin, Germany, 2013.

3. Gharib, G.; Saadeh, R. Reduction of the Self-dual Yang-Mills Equations to Sinh-Poisson Equation and Exact Solutions. WSEAS Interact. Math. 2021, 20, 540-546. [CrossRef]

4. Debnath, L. Nonlinear Partial Differential Equations for Scientists and Engineers; Birkhäuser: Boston, MA, USA, 2005.

5. Sobczyk, K. Stochastic Differential Equations: With Applications to Physics and Engineering; Springer Science \& Business Media: Berlin, Germany, 2001.

6. Tian, D.; Ain, Q.T.; Anjum, N.; He, C.H.; Cheng, B. Fractal N/MEMS: From pull-in instability to pull-in stability. Fractals 2021, 29, 2150030. [CrossRef]

7. Tian, D.; He, C.-H. A fractal micro-electromechanical system and its pull-in stability. J. Low Freq. Noise Vib. Act. Control 2021, 40, 1380-1388. [CrossRef]

8. He, C.-H.; Tian, D.; Moatimid, G.M.; Salman, H.F.; Zekry, M.H. Hybrid Rayleigh -Van der Pol-Duffing Oscillator (HRVD): Stability Analysis and Controller. J. Low Freq. Noise Vib. Act. Control 2021. [CrossRef]

9. Widder, D.V. The Laplace Transform; Princeton University Press: London, UK, 1946.

10. Spiegel, M.R. Theory and Problems of Laplace Transforms; Schaums Outline Series; McGraw-Hill: New York, NY, USA, 1965.

11. Agwa, H.A.; Ali, F.M.; K1lıçman, A. A new integral transform on time scales and its applications. Adv. Differ. Equ. 2012, $2012,60$. [CrossRef]

12. Atangana, A. A Note on the Triple Laplace Transform and Its Applications to Some Kind of Third-Order Differential Equation. Abstr. Appl. Anal. 2013, 2013, 1-10. [CrossRef]

13. Dattoli, G.; Martinelli, M.R.; Ricci, P.E. On new families of integral transforms for the solution of partial differential equations. Integral Transform. Spéc. Funct. 2005, 16, 661-667. [CrossRef]

14. Bulut, H.; Baskonus, H.M.; Belgacem, F.B.M. The Analytical Solution of Some Fractional Ordinary Differential Equations by the Sumudu Transform Method. Abstr. Appl. Anal. 2013, 2013, 1-6. [CrossRef]

15. Weerakoon, S. The Sumudu transform and the Laplace transform: Reply. Int. J. Math. Educ. Sci. Technol. 1997, $28,159-160$.

16. Srivastava, H.M.; Golmankhaneh, A.K.; Baleanu, D.; Yang, X.-J. Local Fractional Sumudu Transform with Application to IVPs on Cantor Sets. Abstr. Appl. Anal. 2014, 2014,1-7. [CrossRef]

17. Albayrak, D.; Purohit, S.D.; Faruk, U.Ç. Certain inversion and representation formulas for q-Sumudu transforms. Hacet. J. Math. Stat. 2014, 43, 699-713.

18. Yang, X.-J.; Yang, Y.; Cattani, C.; Zhu, M. A new technique for solving the 1-D burgers equation. Therm. Sci. 2017, 21 (Suppl. S1), 129-136. [CrossRef]

19. Ahmed, S.A.; Elzaki, T.M.; Elbadri, M.; Mohamed, M.Z. Solution of partial differential equations by new double integral transform (Laplace-Sumudu transform). Ain Shams Eng. J. 2020, 2020. [CrossRef]

20. Sullivan, D.M. Z-transform theory and the FDTD method. IEEE Trans. Antennas Propag. 1996, 44, 28-34. [CrossRef] 
21. Butzer, P.L.; Jansche, S. A direct approach to the Mellin transform. J. Fourier Anal. Appl. 1997, 3, 325-376. [CrossRef]

22. Makarov, A.M. Application of the Laplace-Carson method of integral transformation to the theory of unsteady visco-plastic flows. J. Eng. Phys. Thermophys. 1970, 19, 94-99.

23. Yu, L.; Huang, M.; Chen, M.; Chen, W.; Huang, W.; Zhu, Z. Quasi-discrete Hankel transform. Opt. Lett. 1998, $23,409-411$. [CrossRef] [PubMed]

24. Ul Rahman, J.; Lu, D.; Suleman, M.; He, J.H.; Ramzan, M. He-Elzaki method for spatial diffusion of biological population. Fractals 2019, 27, 1950069. [CrossRef]

25. Watugala, G.K. Sumudu transform: A new integral transform to solve differential equations and control engineering problems. Int. J. Math. Educ. Sci. Technol. 1993, 24, 35-43. [CrossRef]

26. Khan, Z.H.; Khan, W.A. N-transform properties and applications. NUST J. Eng. Sci. 2008, 1, 127-133.

27. Elzaki, T.M. The new integral transform Elzaki transform. Glob. J. Pure Appl. Math. 2011, 7, 57-64.

28. Maitama, S.; Zhao, W. New integral transform: Shehu transform a generalization of Sumudu and Laplace transform for solving differential equations. arXiv 2019, arXiv:1904.11370. Available online: https:/ / arxiv.org/abs/1904.11370 (accessed on 15 October 2021).

29. Cetinkaya, S.; Demir, A.; Sevindir, H.K. Solution of Space-Time-Fractional Problem by Shehu Variational Iteration Method. Adv. Math. Phys. 2021, 2021, 1-8. [CrossRef]

30. Saadeh, R.; Qazza, A.; Burqan, A. A new integral transform: Ara transform and its properties and applications. Symmetry 2020, 12, 925. [CrossRef]

31. Qazza, A.; Burqan, A.; Saadeh, R. A New Attractive Method in Solving Families of Fractional Differential Equations by a New Transform. Mathematics 2021.

32. Burqan, A.; El-Ajou, A.; Saadeh, R.; Al-Smadi, M. A new efficient technique using Laplace transforms and smooth expansions to construct a series solutionsto the time-fractional Navier-Stokes equations. Alex. Eng. J. 2021, in press.

33. Saadeh, R. Numerical algorithm to solve a coupled system of fractional order using a novel reproducing kernel method. Alex. Eng. J. 2021, 60, 4583-4591. [CrossRef]

34. Saadeh, R. Numerical solutions of fractional convection-diffusion equation using finite-difference and finite-volume schemes. J. Math. Comput. Sci. 2021, 11, 7872-7891. 